\title{
Le territoire : un dispositif opératoire révélé par l'activité de travail
}

The territory: an operative dispositive revealed by the work activity

\section{Liliana Cunha et Marianne Lacomblez}

\section{OpenEdition}

\section{Journals}

Édition électronique

URL : https://journals.openedition.org/activites/6925

DOI : $10.4000 /$ activites.6925

ISSN : 1765-2723

Éditeur

ARPACT - Association Recherches et Pratiques sur les ACTivités

Référence électronique

Liliana Cunha et Marianne Lacomblez, «Le territoire : un dispositif opératoire révélé par l'activité de travail », Activités [En ligne], 18-2 | 2021, mis en ligne le 15 octobre 2021, consulté le 07 avril 2022. URL : http://journals.openedition.org/activites/6925 ; DOI : https://doi.org/10.4000/activites.6925

Ce document a été généré automatiquement le 7 avril 2022.

\section{c) (†) $\odot$}

Activités est mis à disposition selon les termes de la licence Creative Commons Attribution - Pas d'Utilisation Commerciale - Pas de Modification 4.0 International. 


\title{
Le territoire : un dispositif opératoire révélé par l'activité de travail
}

The territory: an operative dispositive revealed by the work activity

\author{
Liliana Cunha et Marianne Lacomblez
}

\section{NOTE DE L'ÉDITEUR}

Article soumis le 20/04/2021, accepté le 30/08/2021

\section{Introduction}

1 Les relations entre territoire et travail restent, encore aujourd'hui, peu explorées du point de vue de l'activité du travail. Des approches résistent néanmoins à cette forme de déterritorialisation (Boudra, Béguin, Delecroix, \& Pueyo, 2019), car il est vrai que «l'ergonomie de l'activité nous conduit sur le terrain de la réflexion (et de l'action) quant à un développement humain durable dont le référentiel tend davantage à se "localiser" qu'à se "globaliser" " (Lacomblez, cité par Cunha 2012, p.382). Et c'est bien le lieu que nous revendiquons ici : celui d'un territoire qui soit le réceptacle d'un projet de vie et de conditions de travail pour ceux qui y exercent une activité (Lautier, 1999) ; un territoire qui serait « un espace vital », selon Leroi-Gourhan (1965, p. 182), un espace de vie, selon Schwartz, où ont droit de cité les débats de valeurs et de construction des normes du vivre ensemble (Schwartz, $2000 ; 2020$ ).

2 Mais comment l'activité de travail participe-t-elle, concrètement, aux processus de territorialisation? Et comment s'exprime-t-elle dans l'intermédiation entre le niveau «micro" d'analyse, auquel elle adhère, et le niveau «macro» des décisions de l'administration territoriale? Puisque, évidemment, ce que le point de vue de l'activité permet de mettre en exergue, convoque toujours ce que des instances régionales, 
nationales, voire supranationales ont cherché à valoriser dans leur conception de l'organisation et de la dynamique des territoires qui les intègrent. C'est pourquoi, les niveaux «micro» et "macro» ne se correspondent pas en seules "couches» d'analyses se superposant: nous les entendons comme interagissant, dans ce que chacun révèle de l'autre, dans une dialectique qui tend à la régulation.

3 L'activité qui nous a incitées à problématiser notre approche s'inscrit dans le secteur des transports collectifs routiers de passagers. Dans la perspective qui est la nôtre, il convenait donc de ne point oublier que le droit à la mobilité est un impératif du Marché interne (dit auparavant Marché commun), inscrit dans le cadre de la politique commune des transports prévue, actuellement, dans les articles 90 et suivants du Traité sur le Fonctionnement de l'Union Européenne. Justifié par le fait qu'il constitue un besoin collectif, d'intérêt général, il va de soi que la concrétisation de ce droit exigera toujours des politiques publiques. Il relève en fait d'« une sorte de droit "générique" qui conditionne l'existence réelle de la plupart des autres droits » qui lui sont associés (Ascher, 2010, p. 197). En réalité, ce «droit générique » est tenu d'assurer l'accès et la participation de tou.te.s aux activités essentielles de la vie collective, comme le sont celles relevant de l'éducation, des soins de santé, des activités de travail. Il constitue dès lors une condition d'inclusion sociale et de citoyenneté. D'ailleurs, c'est parce que les ressources nécessaires à la vie sociale sont inégalement réparties dans l'espace, et que les individus n'ont pas tous les mêmes chances d'y accéder, que l'action publique dans ce domaine s'impose.

\section{L'entrée sur le territoire...}

4 L'étude de cas que nous avons choisi de privilégier ici a été réalisée dans le cadre du projet de refonte du réseau de transports publics en bus d'une zone de la région Centre du Portugal - un territoire caractérisé comme étant de faible densité populationnelle et « majoritairement rurale » (Cunha, 2012 ; Cunha \& Lacomblez, 2012).

5 La demande avait été formulée par l'entreprise privée qui détient le monopole des concessions d'exploitation du transport routier de passagers sur ce territoire. Avec le soutien de la Mairie, le projet a été attribué à une équipe spécialisée dans la conception de mobilités, composée de trois ingénieurs, une géographe et un mathématicien équipe à laquelle nous avons été associées. La finalité de cette étude était définie dans une articulation de l'amélioration des conditions de mobilité et de la continuité économique de l'entreprise concessionnaire (Cunha, 2012).

6 Il faut ajouter que l'adaptation aux besoins de la population de l'offre de services de transports publics, en milieu rural et de faible densité, au Portugal, intègre les mesures prioritaires de promotion de la cohésion territoriale et de garantie d'accès aux services d'intérêt général (loi n 99/2019 du 5 septembre).

7 Soulignons encore que le territoire ici en question réunit des conditions peu favorables au compromis entre une amélioration des conditions de mobilité et une durabilité économique des entreprises concessionnaires, en raison, précisément, de sa faible densité de population (environ 38,2 habitants $/ \mathrm{km}^{2}$ ) et de sa vaste superficie (approximativement $250 \mathrm{~km}^{2}$ ). Il est à noter aussi qu'une grande partie du territoire est occupée par des bois de pins, mais surtout d'eucalyptus, dont l'exploitation correspond à la principale activité économique des habitants (Cunha, 2012). 


\section{3. ... et sur son réseau de transport collectif} soient marquées par :

1. Une offre restreinte - limitée à deux trajets par jour, sur chaque ligne de bus, en début et en fin de journée - coïncidant plus ou moins aux horaires scolaires, et également limitée aux jours ouvrables et en dehors des vacances scolaires ;

2. Une absence d'interface entre les différentes lignes de bus (configuration radiale du réseau de transport, privilégiant l'accès au pôle le plus important de la région);

3. Une absence d'offre pour les déplacements répondant aux horaires de travail pratiqués par les entreprises de la région (industrie du bois et de la métallurgie, principalement);

4. Un renforcement de l'offre de transport le jour de la foire hebdomadaire.

Les lacunes de cette offre de transports n'échappèrent pas aux responsables de la Mairie. Depuis quelque temps, ils avaient fait l'option de la renforcer par la mise à disposition directe de certains services, notamment afin de garantir la participation des élèves de l'enseignement primaire à des activités extra-scolaires, mais aussi afin d'assurer des déplacements moins réguliers, requis par des collectifs sociaux et sportifs.

Notre insertion dans l'équipe engagée dans le projet de reconception du réseau a aiguisé notre volonté de démontrer les potentialités du point de vue de l'activité l'orientant, d'abord, sur l'analyse de l'activité des concepteurs de mobilité, et l'articulant progressivement, au cours des 18 mois de notre participation à cette étude, sur celle des chauffeurs, multipliant dès lors observations en situations réelles et entretiens, en cours d'activité ou non.

11 En première analyse, ce sont les perspectives contrastées concernant le territoire et son développement qui ont été les plus flagrantes. Car, si les concepteurs avancent un diagnostic de la dynamique de l'offre et de la demande des services de transport en considérant le périmètre du réseau de bus comme le territoire de travail des conducteurs ; les conducteurs, quant à eux, redéfinissent ces limites en inscrivant, dans l'exercice de l'activité et dans son analyse, d'autres lieux dans le tracé du réseau, tenant compte, opérationnellement et explicitement, de la nécessité d'une réduction de la distance d'accès de certaines personnes au service de transport.

\subsection{Quel territoire l'analyse de l'activité des concepteurs de mobilité nous donne-t-elle à voir ${ }^{1} . .$.}

12 La «sensibilité au territoire" (Reis, 2005) suppose d'emblée de questionner des catégories stables de définition de l'espace - par exemple, la dichotomie urbaine vs rurale - comme s'il s'agissait d'un objet neutre, déconnecté du réel et du point de vue de celui qui y vit, y travaille et l'analyse.

Les concepteurs prennent, de fait, le territoire comme un instrument d'analyse. Selon eux, le territoire existe objectivement et peut être facilement appréhendé, décrit et expliqué. La "sensibilité au territoire", dans ce cas, se traduit à travers le recueil de plusieurs indicateurs tangibles, tenus comme étant les références essentielles dans la prise de décision concernant la réorganisation du tracé des lignes de transport, les arrêts, ou les temps de déplacements entre ceux-ci. 
14 Approché de cette façon, le territoire est, si on reprend Latour (2005), « a matter-offact ", une matière qui s'explique d'elle-même - dans des limites préalablement définies et un cadre d'analyse formellement établi ${ }^{2}$. Il est caractérisé à partir d'un ensemble d'indicateurs de référence communs, utilisés dans la comparaison avec d'autres territoires, tels que : la densité de population; la localisation et les types de pôles générateurs de mobilité (p.ex. emplacement des services essentiels; groupes d'établissements scolaires ; infrastructures d'aide sociale destinée à la communauté) ; et les matrices origine-destination des déplacements. Pour la réalisation de ces matrices, les concepteurs ont mené un suivi de l'activité des chauffeurs en privilégiant les décomptes du nombre de passagers à chaque arrêt du bus, avec enregistrement de ce qu'ils désignaient comme «irrégularités » - par rapport aux itinéraires empruntés, aux lieux des arrêts ${ }^{3}$ et aux horaires de circulation ${ }^{4}$.

Ces indicateurs ont soutenu la construction de cartes qui les représentent. L'utilisation de la cartographie et des systèmes d'information géographique (SIG) sont, dans ce cas, les principaux supports de connaissance et de représentation du territoire (cf. Figures 1 et 2).

Figure 1 : Représentation du territoire en fonction des variations de densité de population. Figure 1: Representation of the territory according to the variations in population density

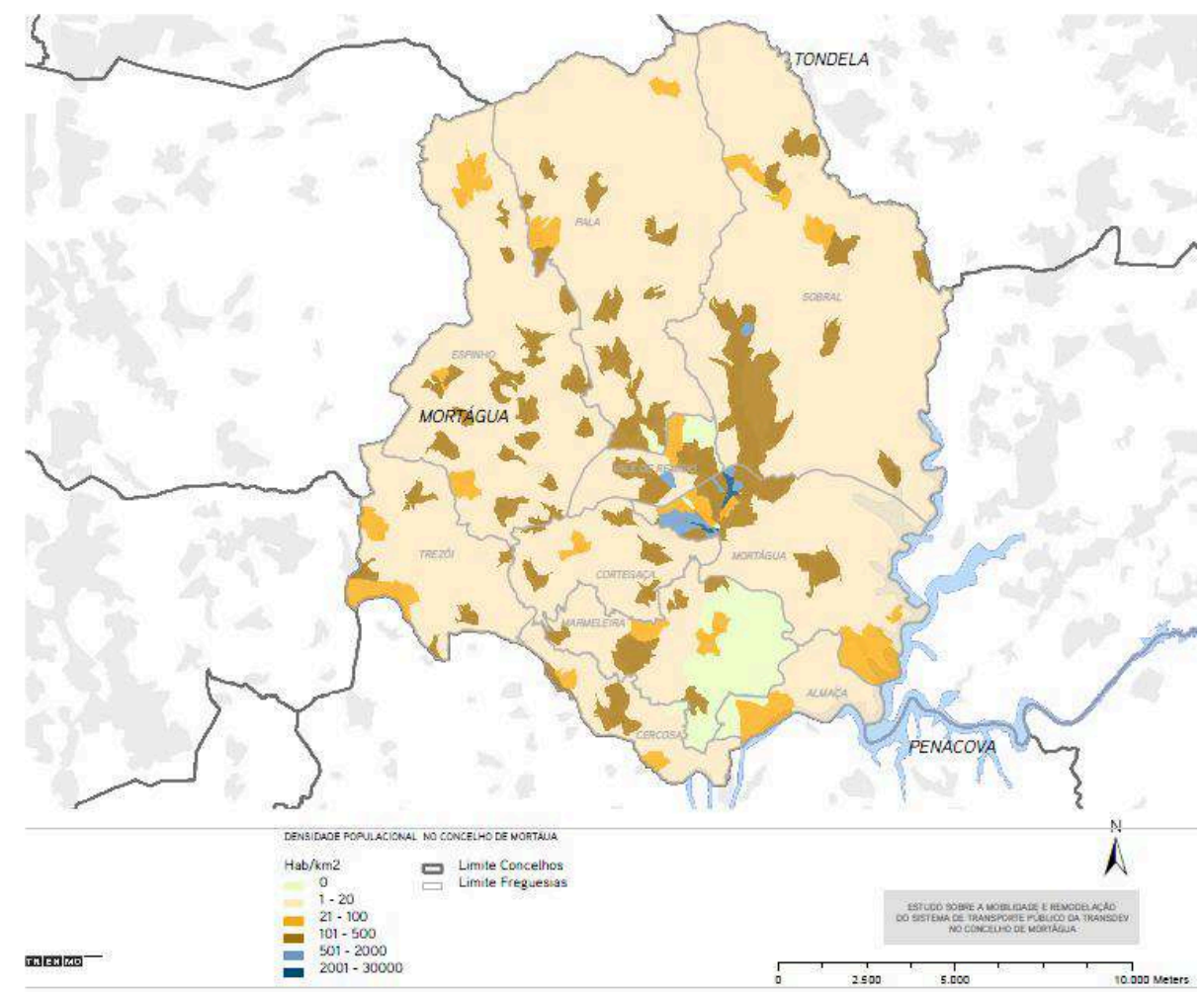


Figure 2 : Représentation du territoire selon les pôles générateurs de mobilité (ex : écoles, centres de santé...).

Figure 2 : Representation of the territory according to mobility generator poles (e.g., schools, health center providers...)

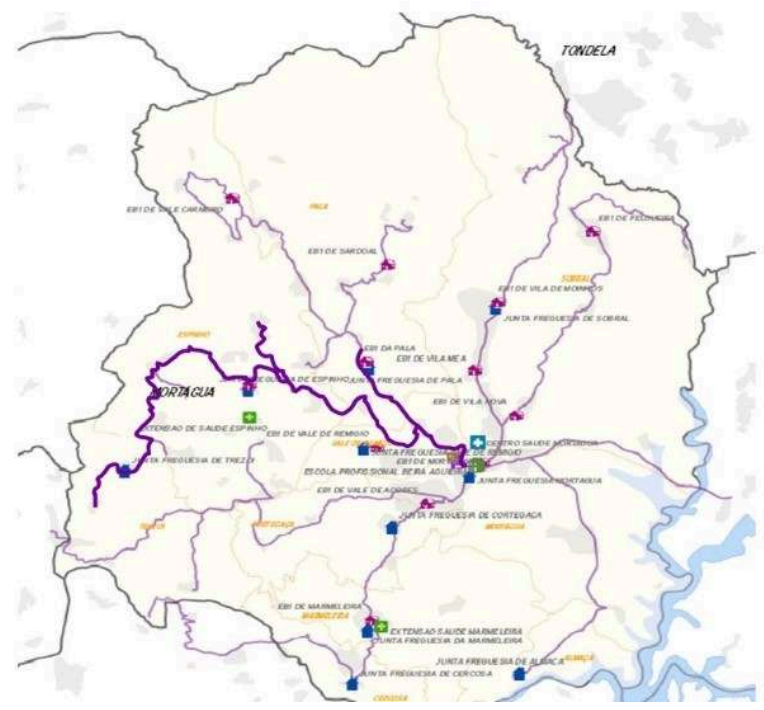

La proposition du nouveau réseau assurait une plus grande couverture spatiale des services prestés (25,5 $\mathrm{km}$ de plus que le réseau précédent), l'introduction d'un nouveau circuit lors d'un jour particulier (le mardi) avec accès au siège administratif de la commune, et l'intégration de quelques-uns des circuits spéciaux assurés par la Mairie.

\section{2. ... et que dit-elle, ou non, de l'activité des chauffeurs ?}

L'équipe des concepteurs s'attarde peu à l'activité des chauffeurs, perçue, paradoxalement, à la fois comme indispensable et résiduelle dans une approche essentiellement normative. Les conditions d'emploi de ces chauffeurs n'ont pas été davantage objet de réflexion de leur part. Or, malgré le fait que la Mairie a la responsabilité de la régulation de l'offre des services de transport, les conditions de travail et d'emploi des chauffeurs sont définies par l'entreprise détentrice de la concession d'exploitation. L'équipe gagnait donc à être interpellée sur ce point.

En réalité, les horaires d'activité des conducteurs sur ce territoire sont "coupés", définis par des trajets qui sont en aller-retour et associés à des situations contractuelles très variables - souvent marquées par la précarité des contrats de travail, fréquemment à temps partiel, et légitimées par le fonctionnement du réseau restreint à deux circulations quotidiennes, sur chaque ligne, et en période scolaire. C'était le cas de l'un des rares chauffeurs « à temps plein » ayant travaillé sur ce territoire :

« Il y a des contrats là-bas qui sont de 20 heures par semaine, voire moins, il y en a un de 17. Maintenant, si vous considérez la valeur de base [le salaire minimum], vous voyez la valeur qu'ils offrent aux personnes? Et puis il y a les inconvénients de ce partiel qu'ils proposent : c'est un partiel qui occupe presque toute la journée. (...) Il y a là un contrat qui est presque comme "tu gagnes ce que tu travailles", et quand vous êtes à l'arrêt, vous ne gagnez pas. Donc, il a un horaire... mais il a 3 périodes de travail... Alors, voyez... ils sont à l'arrêt durant 4 heures, et ne gagnent pas ».

Et c'est pourtant là une question entendue par les concepteurs comme extrinsèque au projet et à eux-mêmes, en tant que professionnels, même si les conditions de travail et 
d'emploi des chauffeurs constituent un facteur déterminant des alternatives dont peuvent bénéficier les citoyens de ce territoire. L'un d'eux nous dit ainsi :

«La posture que j'adopte est “je suis embauché pour ceci, je n'ai rien à voir avec cela" [les coupures de service ou des horaires]. (...) Dans cet aspect de la solution, j'ai pris mes distances face à l'entreprise, sinon c'était trop douloureux... Pour eux, c'est couper, couper...»

Mais, notre analyse de l'activité des chauffeurs a permis d'aller plus loin dans la compréhension de l'organisation de leur temps de travail, donnant une nouvelle visibilité de leur contribution à la réinterprétation du concept de service public de transport sur ce territoire. Son contenu y est élargi dans une référence plus attentive aux conditions d'accès à la prestation du service. Le «droit à la mobilité » trouve ainsi avec eux un moyen pour sa concrétisation, à travers la volonté de garantir une réelle équité territoriale.

\subsection{Comment l'activité de travail produit-elle le territoire?}

Pour illustrer le contraste entre l'approche du territoire propre aux concepteurs et celle que traduit l'activité de travail des conducteurs, nous prendrons l'exemple de la ligne de bus - «Meligioso - Mortágua »-identifiée par un trait plus prononcé sur la Figure 2, et mise en évidence dans la Figure 3.

Soulignons que ce territoire est, à la fois, le territoire de travail et de vie de ces conducteurs. Ils vivent dans la commune où ils travaillent et en sont légitimement des acteurs. Contrairement à ce qui se passe dans des lieux à densité de population plus élevée, ou dans des réseaux de transport où les chauffeurs alternent entre différentes lignes, ici ils circulent toujours sur la même ligne (il y a 9 lignes et 9 conducteurs), ils connaissent bien leurs usagers, leurs noms, le lieu de leur résidence et à quelle distance ils se trouvent de l'arrêt le plus proche. Les savoirs qu'ils détiennent, en tant que citoyens actifs de ce territoire, sont mobilisés dans leur activité, en réponse aux besoins concrets des usagers.

Les observations et entretiens menés avec les chauffeurs ont révélé des stratégies qui sont assumées dans l'activité de travail en vue de réduire la distance d'accès des usagers au réseau de transport, et d'élargir les limites des itinéraires. La réalisation d'écarts sur le tracé de la ligne ainsi que l'arrêt en des endroits non balisés (cf. Figure 3) en sont des exemples - et ils contribuent effectivement à compenser les inégalités d'accès aux transports en commun que le réseau de transport, tel que défini, finissait par reproduire. 
Figure 3 : Représentation d'une ligne du réseau de transport par les concepteurs de mobilité (layer de base) complétée par les traces des détours effectués sur cette ligne, et établis sur le territoire, dans et par l'activité des conducteurs (layer avec les traces de l'activité signalées).

Figure 3 : Representation of a line in the transport network conceived by the mobility designers (base layer) and of the signs of deviations made along this line, and solidified in the territory, in and through the drivers' activity (layer with the signs of the activity)

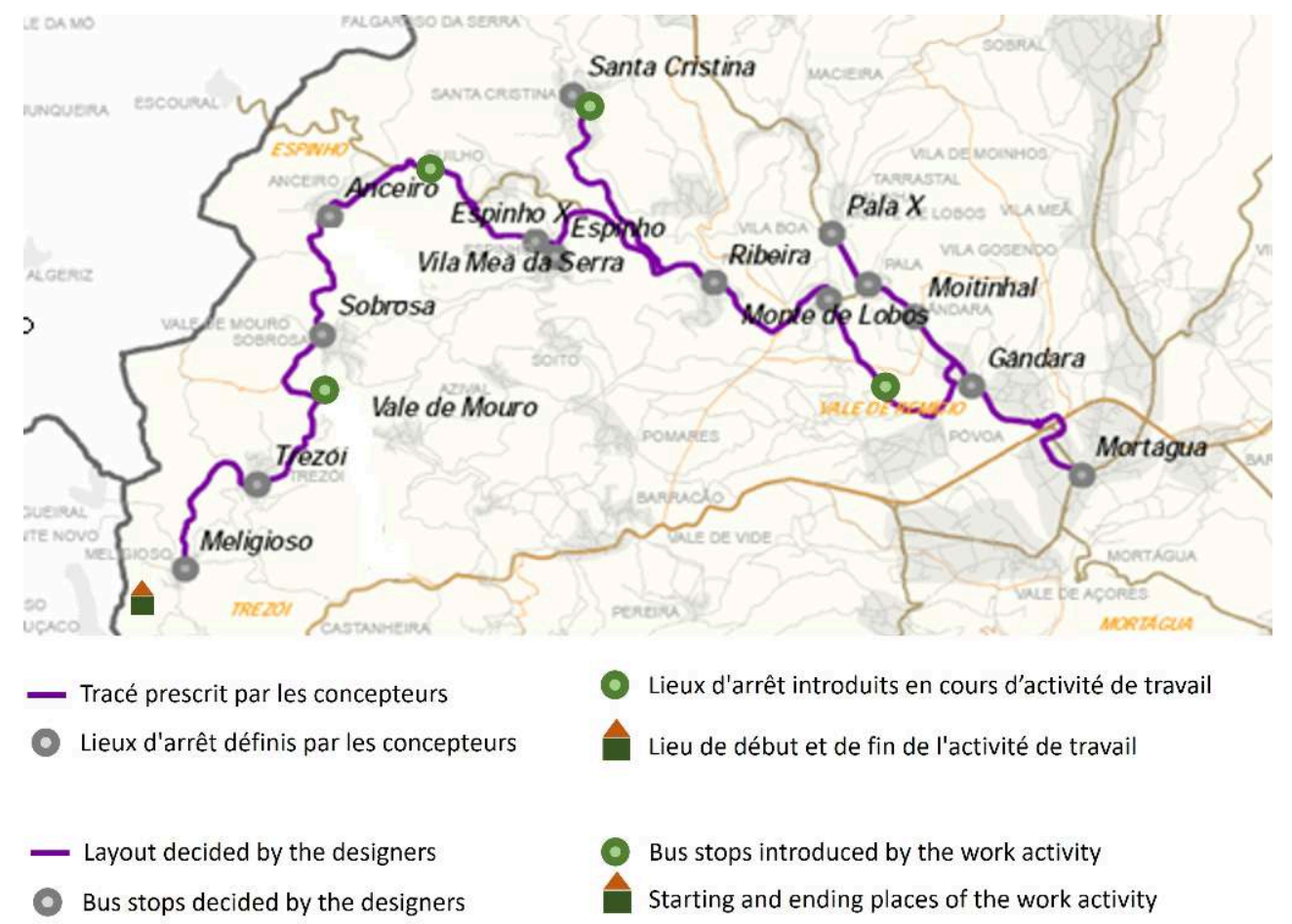

À titre d'exemple, dans le hameau de «Monte de Lobos ", un arrêt est formellement prévu au milieu de l'agglomération - mais, en réalité, ce hameau s'étend sur une distance considérable. La population avait donc rédigé une pétition demandant à la Mairie le placement institué d'un autre arrêt. Cette demande n'ayant pas été accordée, les chauffeurs s'arrêtent cependant toujours à l'endroit revendiqué, en tenant compte du fait que certains passagers vivent parfois à plus de $3 \mathrm{kms}$ d'un arrêt du bus.

Le potentiel de mobilité dont peuvent bénéficier ceux qui utilisent ces transports en commun est ainsi, en partie du moins, la résultante de l'histoire de l'activité de ceux qui fournissent ce service sur ce territoire - et, dès lors, des alternatives sédimentées par leur activité, avec constance et persévérance.

On comprend mieux, alors, combien l'analyse de ces processus de territorialisation permet une ouverture heuristique du concept même de territoire : le territoire n'est plus vu comme un donné, mais il est compris en tant que " processus de vie collective » (Reis, 2007).

C'est le cœur de la distinction entre cette approche des conducteurs et celle des concepteurs - et elle est due, évidemment, au fait qu' "...) ils appartiennent à deux mondes différents et (...) s'ils sont différents, c'est, avant tout, parce que les solutions (...) qu'ils proposent aux problèmes posés sont fonction de la place différente que les uns et les autres occupent (...)» (Oddone, Re, \& Briante, 1981, p. 200).

On dira donc que plus qu'une matter-of-fact, le territoire constitue une matter-ofconcern (Latour, 2005), de débat et de dialectique entre différentes échelles d'analyse (Reis, 2005), dont les enjeux sont multiples. 
Ainsi, la réalisation des détours et l'ajout d'arrêts ne sont-ils pas sans conséquence sur la durée du trajet et sur la programmation du temps de travail des chauffeurs. C'est en fait la raison pour laquelle ils débutent leur journée de travail bien plus tôt, ayant planifié un parcours qui, malgré les détours et arrêts non prescrits, évitera aux élèves les retards à l'école. Quant aux autres usagers, en bons connaisseurs de cette pratique, ils sont présents aux arrêts plus tôt que prévu.

Mais le débat demeure, particulièrement pour ce qui concerne le manque de reconnaissance de l'importance de ces stratégies :

«Il n'y a que des inconvénients sur ce trajet (...) : c'est une route trop étroite et envahie par des camions qui chargent le bois des pinèdes. Et si j'attrape un tracteur, ou un camion, je dois le supporter jusqu'à son arrivée. Cela m'est arrivé plusieurs fois. Parce que c'est comme ça, personne ne peut être inattentif. Et ma plus grande préoccupation, ici à mon travail, c'est que je dois respecter un horaire [l'heure d'arrivée à destination, pour respecter l'horaire scolaire]. (...) C'est pourquoi je quitte la maison bien plus tôt. Par exemple, au lieu de commencer à $7 \mathrm{~h} 00$, je suis là à $6 \mathrm{~h} 30$. On finit par être défavorisé, toujours, de toutes les manières : moi arrivant tôt, et ils ne nous paient pas, mais de plus, si on se fait prendre pour excès de vitesse, cela sortira de notre poche. Bon, mais c'est ce que je dis parfois et ça m'énerve, et après s'ils parviennent à couper 10 minutes aujourd'hui, 10 minutes demain... ».

\section{L'activité de travail et les marqueurs des processus de territorialisation}

31 La discussion sur ces processus de territorialisation soulève des questions d'ordre méthodologique qui lui sont propres: quels outils médiateurs peuvent être utilisés pour appréhender ces dynamiques que révèle l'activité de travail ?

Nous avons essayé de résoudre cette question dans la représentation du territoire telle qu'elle est reprise sur la carte de la Figure 3 - à savoir : en n'attribuant pas d'exclusivité aux indicateurs utilisés par les concepteurs, et en superposant un autre layer où sont représentés les bifurcations, les interactions ou autres formes de sociabilité qui configurent les processus de territorialisation. Ceux-ci se déroulent dans une logique quotidienne, de façon plus ou moins répétitive, en des lieux concrets. Toutefois, ils comportent également une part d'inédit: leur évolution est d'ailleurs souhaitée et le changement des parcours et des arrêts au fil du temps est attendu, notamment au début de chaque année scolaire, en raison de l'entrée de nouveaux élèves à l'école et de la sortie d'autres.

33 Nous reprenons de la sorte les "lieux", dans le sens assumé par les conducteurs: comme points de l'itinéraire où se dessinent les « réserves d'alternatives » (Schwartz, 2000) que l'activité propose face aux asymétries des ressources existantes (ex. : veiller à ne pas dépasser les $3 \mathrm{~km}$ entre le domicile des passagers et un arrêt; trouver une solution aux inconvénients des arrêts sans abri). Et nous identifions ces lieux comme des «marqueurs", physiques ou symboliques, du territoire. Ce sont des "points pivots » qui révèlent l'introduction de changements dans un système prédéfini, en raison de difficultés expérimentées par les opérateurs dans le contrôle de la dynamique d'une situation (De La Garza, 2005) - ici : dans les usages du territoire par les citoyens.

Nous avons ainsi identifié dans la Figure 3, comme «marqueurs physiques » : des arrêts improvisés (ex : près d'un panneau d'affichage, d'une borne postale sur la route, mais 
aussi à la porte d'un café quand l'arrêt prévu n'a pas d'abri pour les passagers); ou encore le lieu où le conducteur commence et termine le trajet (dans le cas repris ici, c'est son domicile, sachant que les conducteurs de ce réseau se voient attribuer la ligne la plus proche de chez eux ${ }^{5}$ ).

Quant aux repères "symboliques ", on donnera ici l'exemple de la gestion par les chauffeurs de l'habitacle du bus : l'absence d'une distinction entre la porte d'entrée et celle de sortie des passagers (il n'y a pas de barrière à l'entrée et les passagers ne montrent pas leur abonnement, car «ce sont des passagers de tous les jours!»); l'existence de sièges «marqués » (par exemple, l'un des chauffeurs a défini un ordre de priorité selon le critère de l'âge : les plus petits enfants sont assis à l'avant, et les plus âgés, jeunes ou adultes, à l'arrière du bus); ou encore, réserver un espace à l'intérieur du bus pour y laisser les cartables (et éviter aux écoliers d'avoir à les porter debout durant tout le trajet). Contrairement à une approche du territoire basée sur des indicateurs prédéfinis, l'identification de ces marqueurs, selon le point de vue de l'activité de travail, révèle de cette façon d'autres lieux qui façonnent le territoire. Ce sont toujours des lieux qui ont émergé de débats et d'une activité normative, ils sont ancrés dans l'activité de travail et sédimentent des interactions. Ce sont, ainsi, des marqueurs d'identités collectives (Banos, 2009).

Envisager le territoire en tant que dispositif opératoire, c'est donc vouloir révéler sa mise à jour permanente, sa maturation quotidienne, sans négliger sa reconnaissance en tant que ressource collective.

\section{Quand le territoire devient une catégorie d'analyse}

La pertinence heuristique du concept de territoire ne lui est pas immanente. Il nous faut définir son statut et sa place dans nos interventions. À quel moment le territoire cesse-t-il d'être simplement le «sol » où l'histoire des activités de travail, et l'histoire de ceux qui en sont les protagonistes, s'enchevêtrent, au quotidien, pour devenir alors une catégorie d'analyse?

a caractérisation du territoire à l'aide des indicateurs spatialisés tels ceux des SIG, nous en dit trop peu sur ce qu'est en vérité le territoire et sur les aspects problématiques que peut révéler le point de vue de l'activité. En contrepartie, en effet, l'analyse de l'activité de travail, lorsqu'elle tient compte de son encastrement territorial, centrée sur la vie quotidienne et sur les interactions entre les citoyens de ce territoire, s'inscrit bien dans une approche multi-niveaux telle que l'exige la bonne compréhension du territoire.

Dans cette perspective, le territoire n'est donc pas une variable indépendante de notre cadre de vie : le jeu de la société entre dans la "production de l'espace» (Lefebvre, 1974). Il s'agit d'un recours collectif à intégrer dans le cours de l'avancement du Bien Commun. Et, si nous partageons une conception de développement territorial endogène, provenant de recours matériels et immatériels, propres à un territoire donné, et provenant des savoirs détenus par les acteurs qui l'occupent, le sens de ce développement ne peut faire abstraction de l'importance des relations travail-territoire dans la conceptualisation du réel (Schwartz, 2008).

Dans le cadre de l'Union Européenne, tant la Politique des transports que la Politique Agricole Commune sont reconnues comme étant des "politiques territoriales 
implicites ", à savoir, des "politiques sectorielles ayant une forte capacité à structurer (sans nécessairement ordonner) le territoire. (...) Des politiques qui, poursuivant des objectifs de nature sectorielle, induisent, directement ou non, des effets très pertinents sur les formes d'occupation et d'organisation du territoire » (Ferrão, 2015, pp. 331-332, traduction libre).

41 Toutefois, comme nous l'avons constaté à partir de l'étude de cas présentée dans cet article, la concrétisation locale de ces politiques publiques ne répond pas toujours aux besoins qui sont censés être couverts. La contribution de l'activité de travail s'est révélée alors, dans l'analyse des « résidus » des politiques de transport collectif, comme un instrument, singulièrement affûté, pour l'approche des formes de ségrégation sociospatiale de l'accès à la mobilité.

42 Le territoire, en assumant la configuration que le point de vue de l'activité révèle, sert alors d'intermédiaire entre les niveaux «macro » et "micro» de l'analyse, entre la conception de ce que doivent être le service public et le droit à la mobilité, et les tentatives de compenser les inégalités dans leur accès. C'est d'une certaine façon, la demande sociale que les protagonistes du territoire adressent au travail : l'ouverture aux « réserves d'alternatives » (Cunha, Lacomblez, \& Schwartz, 2020) dans l'adaptation des politiques publiques aux spécificités locales.

43 La relation territoire-lieu - en assumant le lieu non pas comme une stricte localisation, mais comme un point-pivot, un marqueur matériel ou symbolique des processus de territorialisation - permet de saisir pourquoi et comment l'activité de travail contribue à produire le territoire. Ce sont des marqueurs essentiels pour comprendre ce réel. Le concept de lieu en devient une face visible du territoire et une synthèse concrète des débats de valeurs et de normes d'un collectif, composé de travailleurs et de citoyens.

Dans le cadre de l'étude reprise ici, les résultats et leur discussion avec les concepteurs ont été déterminants pour la refonte du réseau de transport local et l'extension de sa couverture territoriale. Le legs de l'activité, avec ses traces sur le territoire, s'est ainsi inscrit de façon précise dans un projet de développement territorial.

Notre réflexion sur le territoire a exigé une approche, à la fois synchronique et diachronique, qui permette de comprendre la configuration appréhendée aujourd'hui, dans l'épaisseur temporelle qui a permis sa production. C'est dire que l'analyse des processus de territorialisation requiert une démarche continuée dans le temps, une perspective de «temps prolongé » sur les données. De plus, la relation avec le territoire convoque des micro et macro-territorialités, pour lesquelles il convient de différencier les acteurs et les intérêts concernés, ainsi que les modes de régulation définis.

Mais, on l'aura compris, assumer une approche territoriale requiert également une épaisseur temporelle pour l'activité de recherche. Certes, le territoire qui nous a interpellées présente aujourd'hui de nouvelles configurations et de nouvelles questions. Il faudrait donc y retourner et retrouver ses acteurs. Malheureusement, la discussion à propos des politiques de transport dans les endroits à faible densité populationnelle ne fait pas partie de l'agenda actuel des priorités - la prérogative étant attribuée aux questions de changement climatique et de vieillissement de la population (PNPOT, 2019). Il est vrai que les préoccupations concernant le territoire et ses formes de problématisation sont nécessairement contingentes et l'engagement dans la gouvernance territoriale se construit dans les essais de garantie de la meilleure articulation possible entre les différents axes des politiques publiques (Ferrão, 2015). 
Sans oublier, pour autant, que les conditions d'emploi, de travail, d'activité, constituent toujours une question « d'intérêt public ».

\section{BIBLIOGRAPHIE}

Ascher, F. (2010). Les nouveaux principes de l'urbanisme. La Tour d'Aigues : Éditions de l'Aube.

Banos, V. (2009). Réflexion autour de la dimension spatiale des processus normatifs. Géographie et cultures, 72, 80-98.

Boudra, L., Béguin, P., Delecroix, B., \& Pueyo, V. (2019). Taking into account the territory in occupational risks prevention. The case of work activity in waste sorting centers. Le Travail Humain, 82, 99-128.

Cunha, L. (2012). Mobilidades, territórios e serviço público: debates sobre o interesse colectivo à margem do paradigma de uma sociedade móvel. Thèse présentée en vue de l'obtention du grade de Docteur en Psychologie, Porto, FPCE-UP.

Cunha, L., \& Lacomblez, M. (2012). From the "terrain" to "territory": which contributions from mobility and bus drivers' activity towards local development? Work, 41, 6156-6161. https:// doi.org/10.3233/WOR-2012-1077-6156

Cunha, L., \& Lacomblez, M. (2021). Territory as a Construct of Work Activity and an Operative Dispositive for and Through Action. In P. Neumann et al. (Eds.), Human Factors and Ergonomics in a connected world/L'ergonomie 4.0. Cham, Springer [accepted for publication in 2021].

Cunha, L., Lacomblez, M., \& Schwartz, Y. (2020). Traces de l'activité de travail et tracés de son analyse dans deux pays du (des) « Sud(s) » : les débats qui dépassent le rapport entre empirie et théorie. In M. Prévot-Carpentier, L. Paltrinieri \& M. Nicoli (Eds.), Le philosophe et l'enquête de terrain : le cas du travail contemporain (pp. 149-166). Toulouse : Octarès.

De La Garza, C. (2005). Aportes del método de los "puntos pivote" a un estudio prospectivo de seguridad en el campo de la interoperabilidad ferroviaria. Laboreal 1(1).

Ferrão, J. (2015). Ambiente e território : para uma nova geração de políticas com futuro. In V. Soromenho-Marques \& P. Trigo Pereira (Eds.), Afirmar o futuro: políticas públicas para Portugal. Desenvolvimento sustentável, economia, território e ambiente, vol. II (pp. 328-336).

Latour, B. (2005). Reassembling the Social - an introduction to Actor-Network-Theory. New York : Oxford University Press.

Lautier, F. (1999). Ergotopiques : sur les espaces des lieux de travail. Toulouse : Octarès.

Lefebvre, H. (1974). La production de l'espace. L'Homme et la Société. Paris : Anthropos.

Leroi-Gourhan, A. (1965). Le geste et la parole. Paris : Albin Michel.

Oddone, I., Re, A., \& Briante, G. (1981). Redécouvrir l'expérience ouvrière. Paris : Éditions sociales.

PNPOT - Programa Nacional da Política de Ordenamento do Território (2019). Primeira revisão Lei n.․9 99/2019 de 5 de setembro, Diário da República n.ำ170/2019. Lisboa, Portugal : Direção-Geral do Território. 
Reis, J. (2005). Uma epistemologia do território. Estudos - Sociedade e Agricultura, 13(1), 51-74.

Reis, J. (2007). Ensaios de economia impura. Coimbra : Almedina.

Schwartz, Y. (2000). Le paradigme ergologique ou un métier de philosophe. Toulouse : Octarès.

Schwartz, Y. (2008). Revisiter l'activité humaine pour poser les questions du développement. Ergologia, 1, 135-169.

Schwartz, Y. (2020). Activité(s) et usages de soi : quel(s) «milieux » pour l'humain ? Les Études philosophiques, 201, 93-123. https://www.cairn.info/revue-les-etudes-philosophiques-2020-1page-93.htm

\section{NOTES}

1. Ce texte reprend en partie les hypothèses d'analyse, les résultats et les voies d'interprétation présentées dans Cunha et Lacomblez (2021).

2. Une première analyse par les concepteurs du territoire en question dans cette étude de cas, a été réalisée à partir de la nomenclature NUTS (Nomenclature de l'Unité Territoriale à des fins Statistiques), qui identifie différentes catégories territoriales. Cette nomenclature a été introduite par EUROSTAT, en vue d'harmoniser les données statistiques des régions des pays de l'Union Européenne. Ses classifications permettent une description du territoire, fondée sur le principe d'une hiérarchie des différentes catégories géographiques (NUTS I, II et III). Néanmoins, il existe une grande hétérogénéité du point de vue socio-économique entre les territoires qui intègrent la même catégorie d'analyse.

3. Ils essaient d'identifier si les arrêts sont équipés - à savoir : s'ils disposent d'un abri, de bancs et d'un panneau d'information avec une carte de l'itinéraire de la ligne et les horaires.

4. Ces chauffeurs circulaient presque toujours avant le début de l'horaire défini par l'employeur, contrairement à une prémisse tacite, mais informellement partagée dans le secteur, qu'il est toujours plus critique pour les passagers que le bus circule avant l'horaire prévu, plutôt qu'avec quelques minutes de retard. Nous reprendrons cette question plus loin.

5. Le bus est alors ramené au domicile du chauffeur, évitant ainsi à l'entreprise les frais d'un parcours de $35 \mathrm{~km}$ "à vide » (sans entrée ni sortie de passagers) jusqu'au dépôt, situé dans une autre commune, bien que contiguë au territoire en analyse.

\section{RÉSUMÉS}

L'analyse de l'activité de travail permet de repenser le statut du territoire dans nos recherches. Car il n'est pas seulement le «sol» sur lequel l'histoire des activités de travail et de leurs protagonistes est tissée au quotidien: nous considérons en effet que le territoire est aussi un produit de l'activité, il est agi et reconstruit quotidiennement - l'activité intervient dans les processus de territorialisation. Cette perspective justifie que l'activité de travail prenne place dans les débats sur le vivre ensemble et sur les options de l'action publique dans l'aménagement du territoire. On interpelle dès lors les modes de participation publique, en revendiquant que les citoyens travailleurs y soient plus étroitement associés. Mais comment procéder? Quelles traces permettent de démontrer l'apport de l'activité de travail aux processus de territorialisation? Ces 
questions ont émergé au cours d'un programme de recherche - et nous les étayons ici en reprenant l'étude développée dans le secteur du transport collectif routier de passagers en milieu rural. L'analyse du territoire, telle que nous la concevons, est ainsi enrichie par le point de vue de l'activité et par les débats qui en émergent. Mais le territoire que nous révèle ici l'activité est surtout celui que ses protagonistes en font, au cours d'un processus de transformation trop souvent peu visible, sédimenté par nombre de répétitions silencieuses - jusqu'à devenir une ressource collective.

Work activity analysis allows us to rethink the status of territory in our research. It is not only the "ground" on which the history of work activities and their protagonists is daily woven: we consider territory to also be a result of the work activity; it is acted upon and reconstructed on a daily basis - the activity plays a role in the processes of territorialization. This perspective justifies the presence of work activity in debates on the collective life and public action options relating to territorial planning. We therefore question the modes of public participation and demand that working citizens be more closely involved. But how should we proceed? What are the signs capable of demonstrating how work activity contributes to territorialization processes? These questions emerged in the context of a research program - and we underpin them in this paper by revisiting a study carried out on the road passenger transport sector in a rural area. The analysis of territory, as we conceive it, is thus enhanced by considering the point of view of the activity and by the debates that emerge from it. The territory thus revealed by the activity is above all what its protagonists make of it, over the course of a process of transformation that all too often lacks visibility; it gradually takes place through a number of silent repetitions, until it finally becomes a collective resource.

\section{INDEX}

Mots-clés : transport collectif routier de passagers en milieu rural, reconception d'un réseau de transport, processus de territorialisation, relais macro-micro, indicateurs et marqueurs

Keywords : road passenger transport sector in a rural area, redesign of a transport network, territorialization processes, macro-micro relations, indicators and markers

\section{AUTEURS}

\section{LILIANA CUNHA}

Faculdade de Psicologia e de Ciências da Educação da Universidade do Porto. Centro de Psicologia da Universidade do Porto. Rua Alfredo Allen, 4200-135 Porto. lcunha@fpce.up.pt

\section{MARIANNE LACOMBLEZ}

Faculdade de Psicologia e de Ciências da Educação da Universidade do Porto. Centro de Psicologia da Universidade do Porto. Rua Alfredo Allen, 4200-135 Porto. lacomb@fpce.up.pt 